Title : will be set by the publisher

Editors : will be set by the publisher

EAS Publications Series, Vol. ?, 2009

e-mail: m.ashley@unsw.edu.au

\title{
PLATO-A ROBOTIC OBSERVATORY FOR THE ANTARCTIC PLATEAU
}

M. C. B. Ashley ${ }^{1}$, G. Allen ${ }^{2}$, C. S. Bonner ${ }^{1}$, S. G. Bradley ${ }^{3}$, X. Cui $^{4}$,

J. R. Everett ${ }^{1}$, L. Feng ${ }^{5}$, X. Gong ${ }^{4}$, S. Hengst ${ }^{1}$, J. Hu$^{6}$, Z. Jiang ${ }^{6}$,

C. A. Kulesa ${ }^{7}$, J. S. Lawrence ${ }^{9,8,1}, \mathrm{Y}_{\text {. Li }}{ }^{10}$, D. M. Luong-Van ${ }^{1}$,

M. J. McCaughrean ${ }^{11}$, A. M. Moore ${ }^{12}$, C. Pennypacker ${ }^{13}$, W. Qin ${ }^{10}$,

R. Riddle ${ }^{14}$, Z. Shang ${ }^{15}$, J. W. V. Storey ${ }^{1}$, B. Sun ${ }^{10}$, N. Suntzeff ${ }^{16}$,

N. F. H. Tothill ${ }^{11}$, T. Travouillon ${ }^{14}$, C. K. Walker ${ }^{7}$, L. Wang ${ }^{5,16}$, J. Yan ${ }^{5}$, H. Yang ${ }^{10}$, J. Yang ${ }^{5}$, D. G. York ${ }^{17}$, X. Yuan ${ }^{4}$, X. Zhang ${ }^{5}$, Z. Zhang ${ }^{10}$, $\mathrm{X}$. Zhou ${ }^{6}$ and Z. Zhu ${ }^{5}$

\footnotetext{
${ }^{1}$ School of Physics, University of New South Wales, NSW 2052, Australia

2 Solar Mobility Pty Ltd, Thornleigh, NSW 2120, Australia

${ }^{3}$ Physics Department, University of Auckland, Auckland 1142, New Zealand

${ }^{4}$ Nanjing Institute of Astronomical Optics and Technology, Nanjing 210042, China

${ }^{5}$ Purple Mountain Observatory, Nanjing 210008, China

${ }^{6}$ National Astronomical Observatories, Chinese Academy of Sciences, Beijing 100012, China

7 Steward Observatory, University of Arizona, Tucson, AZ 85721, USA

8 Department of Physics and Engineering, Macquarie University, NSW 2109, Australia

${ }^{9}$ Anglo-Australian Observatory, NSW 1710, Australia

${ }_{10}$ Polar Research Institute of China, Pudong, Shanghai 200136, China

${ }^{11}$ School of Physics, University of Exeter, Exeter, EX4 4QL, UK

12 Caltech Optical Observatories, California Institute of Technology, Pasadena, CA 91125, USA

13 Lawrence Berkeley National Laboratory, University of California, Berkeley, CA 94720, USA

14 Thirty Meter Telescope Project, Pasadena, CA 91107, USA

15 Tianjin Normal University, Tianjin 300074, China

${ }^{16}$ Physics Department, Texas A\&M University, College Station, TX 77843, USA

17 Department of Astronomy and Astrophysics and The Enrico Fermi Institute, University of Chicago, Chicago, IL 60637, USA
} 


\begin{abstract}
PLATO is a fully-robotic observatory designed for operation in Antarctica. It generates its own electricity (about $1 \mathrm{~kW}$ ), heat (sufficient to keep two 10-foot shipping containers comfortably above $0^{\circ} \mathrm{C}$ when the outside temperature is at $\left.-70^{\circ} \mathrm{C}\right)$, and connects to the internet using the Iridium satellite system (providing $\sim 30 \mathrm{MB} /$ day of data transfer). Following a successful first year of operation at Dome A during 2008, PLATO was upgraded with new instruments for 2009.
\end{abstract}

\title{
1 Introduction
}

PLATO is a robotic observatory designed for the high Antarctic plateau (Lawrence et al. 2009, Luong-Van et al. 2008, Lawrence et al. 2008), and is a descendent of the AASTINO observatory that operated at Dome C during 2003-4 (Lawrence et al. 2005). PLATO was installed at Dome A in January 2008 by a tractor traverse operated by the Polar Research Institute of China and with 17 expedition members, including two astronomers: Zhou Xu and Zhenxi Zhu.

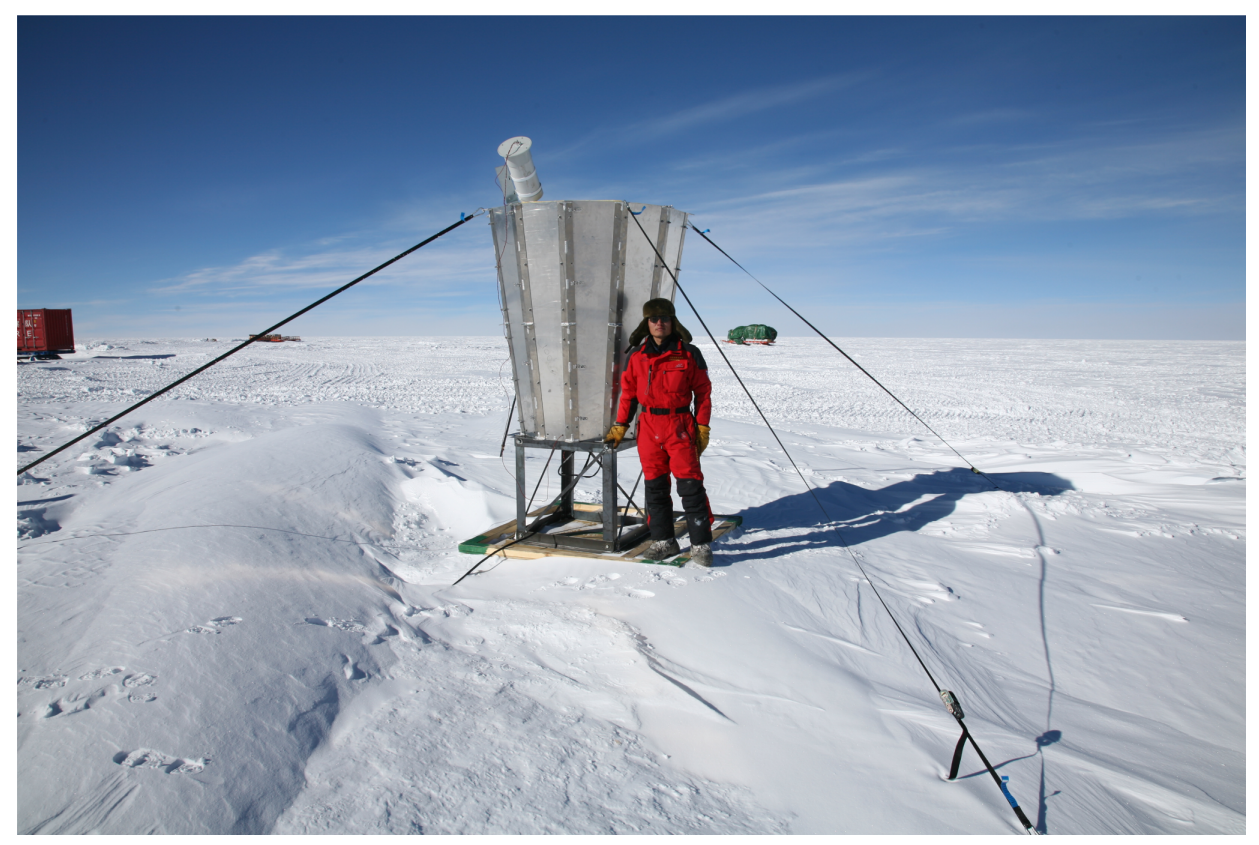

Fig. 1. Xuefei Gong standing in front of the newly installed Snodar, January 2009. Inside the aluminium shell is a $0.9 \mathrm{~m}$ diameter parabolic reflector and compression driver for sending and receiving $\sim 5 \mathrm{kHz}$ sound waves to probe the turbulence in the lowest $200 \mathrm{~m}$ of the atmosphere. The white tube at the top houses a webcamera to allow inspection of the snow coverage on the parabolic reflector. Heaters can be used to remove the snow. 
PLATO operated for 204 days during 2008, finally stopping in early August due to a failure of the flexible exhaust tube from one of the diesel engines, which allowed the engine compartment to fill with exhaust gasses and hence overheat. Details of the engine system are given by Hengst et al. 2008 and Hengst et al. 2009. The original instruments, and results from 2008, are described by Yang et al. 2009.

PLATO was designed to have its engines swapped-out each year, and this was accomplished for the first time in January 2009 by another traverse by the Polar Research Institute of China. This traverse was a very substantial undertaking consisting of 28 people (one of whom, Xuefei Gong, was an astronomer), 4 tractors, 7 PistenBullys, and 44 sleds carrying a total of 570 tonnes. While at Dome A, the team began construction of Kunlun Station, at latitude of $-80^{\circ} 25^{\prime} 08.4^{\prime \prime}$, longitude $77^{\circ} 06^{\prime} 58^{\prime \prime} \mathrm{E}$, height $4087 \mathrm{~m}$, and about $200 \mathrm{~m}$ from PLATO.

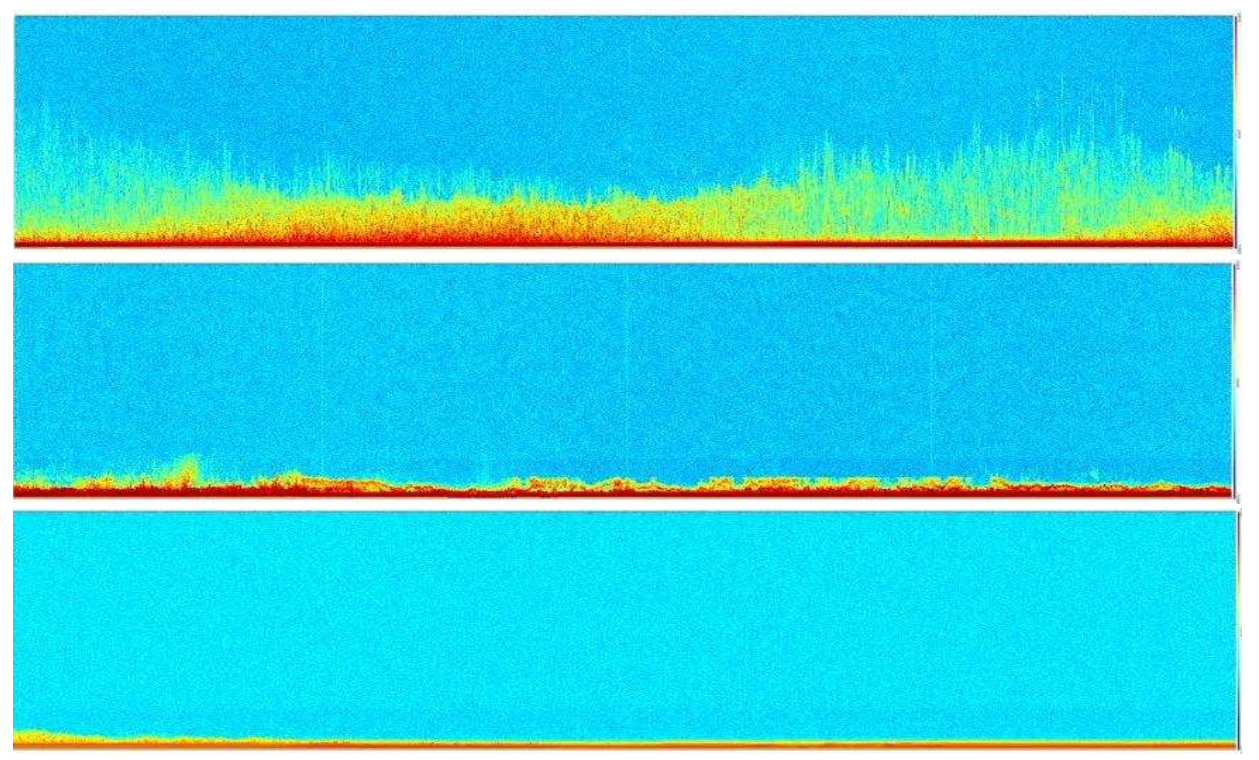

Fig. 2. Three days of Snodar data from 2009. Each plot shows 24 hours on the horizontal axis, and 0 to $180 \mathrm{~m}$ in height above the snow on the vertical axis. The colour indicates the strength of the return echo: red is strongest, and blue is at the ambient noise level. The top plot shows a diurnal cycle that is often visible in summertime, with periods of weaker, more extended, turbulence at local noon. The middle plot is wintertime data showing fine structures revealed by Snodar's $0.9 \mathrm{~m}$ vertical resolution. The lower plot is typical of much of winter, with no turbulence detected above a thin boundary layer; initial analysis shows that the layer is $<20 \mathrm{~m}$ in height for $70 \%$ of the time. As of 2009 May 6, the two Snodars in PLATO had returned $2.3 \times 10^{6}$ individual measurements of the vertical turbulence profile. 

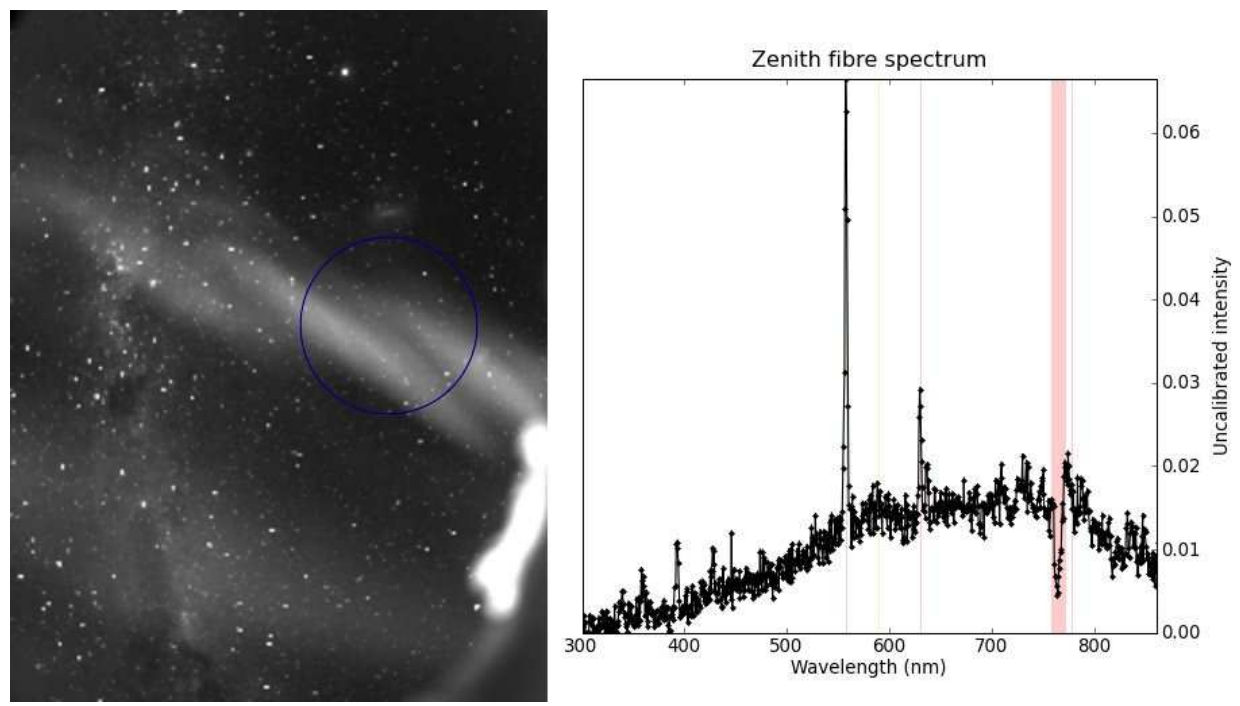

Fig. 3. At left is a V-band image from the Gattini camera taken during the 2009 winter, binned $8 \times 8$, showing a field-of-view $\sim 90^{\circ}$ towards the south celestial pole. The Milky Way and both Magellanic Clouds are visible, as is a prominent aurora; the bright patch at lower right is moonlight reflected from snow on the edge of the instrument window. The blue circle shows the approximate size and position of the area of sky sampled by the Nigel zenith fibre. The plot at right is an uncalibrated spectrum from this fibre taken simultaneously with the image. Bright auroral lines are visible: OI at 557, 630, and $777 \mathrm{~nm}$. The trough at $760 \mathrm{~nm}$ is due to $\mathrm{O}_{2}$ in the atmosphere.

\section{Improvements made during January 2009}

The servicing mission was an opportunity for upgrading PLATO and adding new experiments. For 2009 a new Snodar acoustic radar was deployed (Figs 1 and 2), and the original Snodar (Bonner et al. 2008, Bonner et al. 2009) was upgraded with new electronics. The new Snodar samples every 10 seconds at 4,5 , and $6 \mathrm{kHz}$ to a height of $180 \mathrm{~m}$. The original Snodar samples every 5 seconds at $5 \mathrm{kHz}$ to $120 \mathrm{~m}$. The two Snodars are $20 \mathrm{~m}$ apart, and show remarkable agreement.

CSTAR (Yuan et al. 2008) received upgraded disk storage units. The original two Gattini cameras (Moore et al. 2008) were replaced with a single wide-field optical camera with four filters (Figs 3, 4, and 5). The fuel, oil, and exhaust systems for the engines were improved. The engines and batteries were replaced. New webcameras were added to monitor snow on the Snodar dishes, and to allow the inside of PLATO's Engine Module to be inspected. A microphone was placed in the Engine Module to assist with diagnosing engine faults.

Nigel, a fibre-fed optical spectrograph measuring the sky background from 300 to $850 \mathrm{~nm}$ in three $20^{\circ}$ diameter patches of sky, was installed (Kenyon et al. 2006). Example data from Nigel is shown in Figs 3 and 4. 

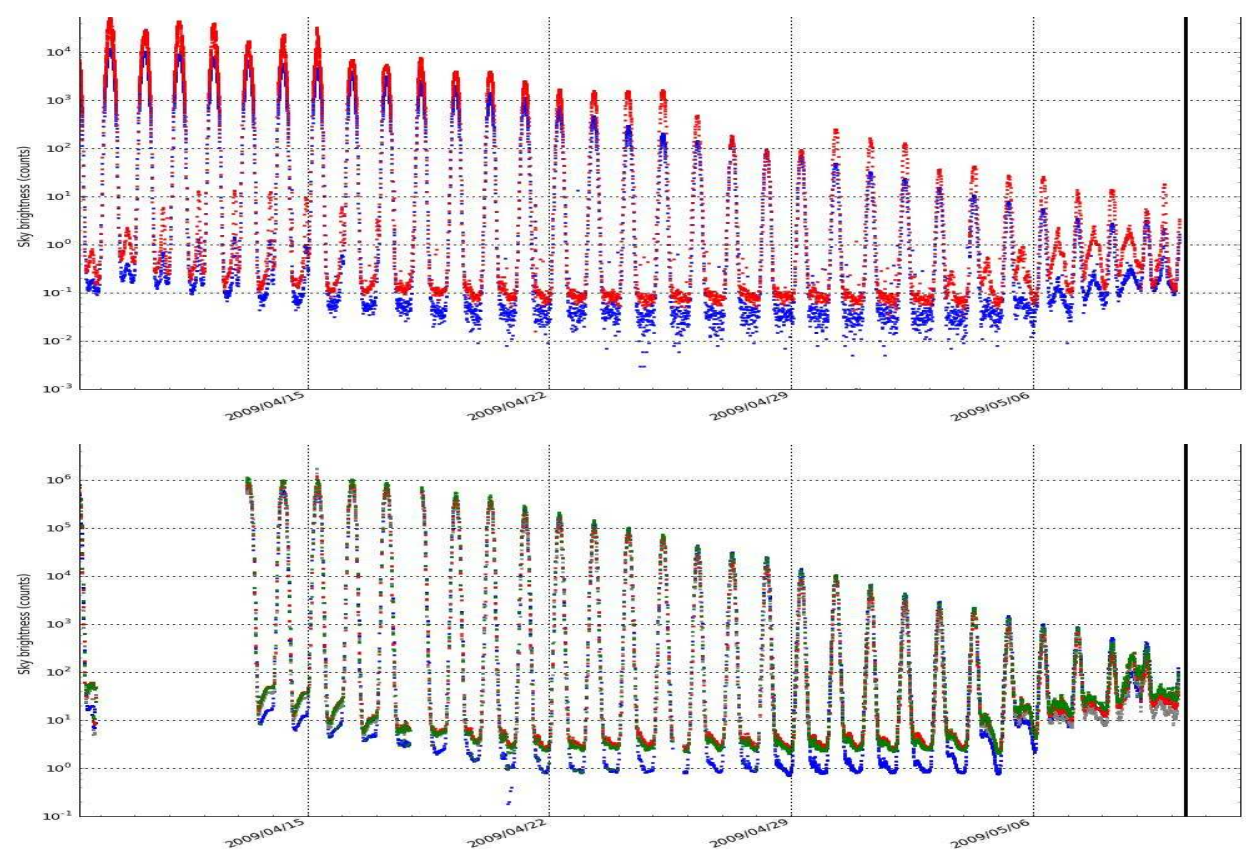

Fig. 4. A month of sky brightness data from Nigel (upper) and Gattini (lower), showing a factor of $10^{6}$ change in the sky brightness depending on the zenith distance of the sun (diurnal fluctuations) and the moon (brightening from early May 2009). The red and blue curves in the upper plot are the integrated intensity from spectra centred on 850 and $350 \mathrm{~nm}$ respectively. The blue, grey, red and green curves in the lower plot correspond to median sky estimates through B, 'OH' (700nm long-pass), R, and V filters respectively. The plots assume a linear CCD response, and have not been flux-calibrated.

A mechanical failure in a gear mechanism in Pre-HEAT (Kulesa et al. 2008) has reduced the functionality of this instrument.

\section{Conclusion}

At the time of writing (2009 August 21), PLATO has been running for 221 days during 2009. The two Snodars, Gattini, Nigel, the CSTAR telescopes, and the webcameras are all returning excellent data. The latest information on PLATO is on the project website at http://mcba11.phys.unsw.edu.au/ plato.

Planning is now under way for the 2009/10 servicing mission, additional instruments, and an enhanced version of PLATO to provide more power, thereby allowing larger instruments to be supported.

We thank the Polar Research Institute of China for making our productive collaboration possible, and the members of the 2008 and 2009 Chinese traverse teams for their efforts installing and servicing PLATO. We acknowledge funding from the Chinese PANDA International Polar Year project, the Chinese Academy 

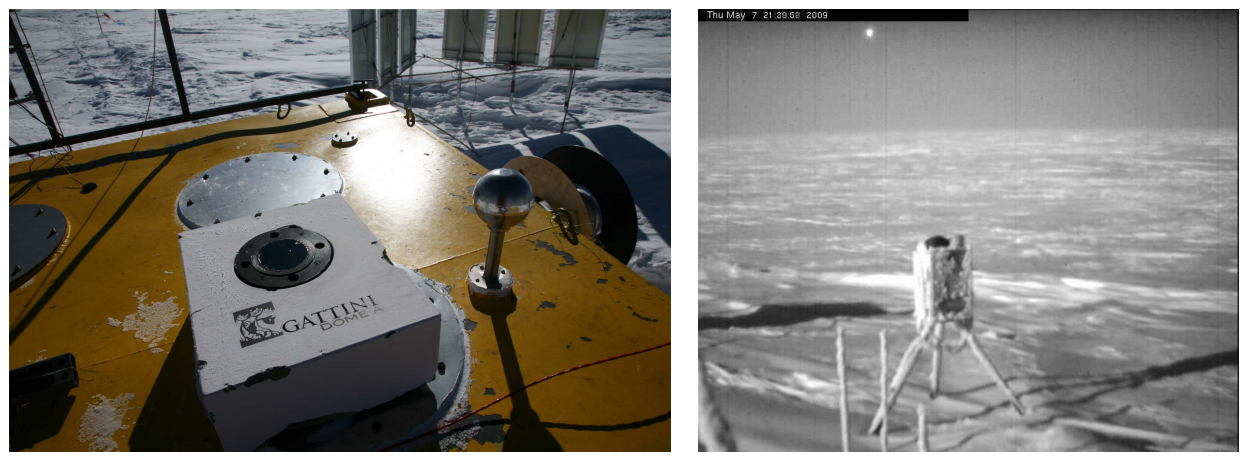

Fig. 5. At left is the Gattini optical camera beneath the white enclosure on the yellow roof of PLATO's Instrument Module. The circular window has an indium tin oxide coating to assist with snow removal. The Nigel optical fibres are mounted within the stainless steel sphere to the right of Gattini. Small electrical heaters are placed close to each fibre, inside the sphere, and require just a few watts to keep the fibres ice-free. Six solar panels, providing about $200 \mathrm{~W}$ of electrical power each, are visible towards the upper right. The photograph at right shows the CSTAR telescopes and tripod as recorded by one of PLATO's eight webcameras on 2009 May 7 at 10 hours UTC. The shadows are cast by the $97 \%$-full moon. The bright object at top left is Jupiter. CSTAR is $\sim 2 \mathrm{~m}$ tall, and its four $0.145 \mathrm{~m}$ telescopes are pointing away from the webcamera in this photograph.

of Science, the National Natural Science Foundation of China, the US National Science Foundation, the Australian Antarctic Division, and the Australian Research Council through the Discovery Projects and Linkage International schemes.

\section{References}

Bonner, C. S. et al. 2008, SPIE, 7012, 70146I-1-70146I-7

Bonner, C. S. et al. 2009, Acoustics Australia, 37, 47-51

Hengst, S. et al. 2008, SPIE, 7012, 70124E-1-70124E-10

Hengst, S., Luong-Van, D. M., Everett, J. R., Lawrence, J. S., Ashley, M. C. B., Castel, D., and Storey, J. W. V., 2009, International Journal of Energy Research, in press

Kenyon, S. L. et al. 2006, SPIE, 6267, 62671M-162671M-9

Kulesa, C. A. et al. 2008, SPIE, 7012, 701249-1-701249-11

Lawrence, J. S., Ashley, M. C. B. \& Storey, J. W. V., 2005, Aust. J. Electrical and Electronics Engineering, 2, 1-12

Lawrence, J. S. et al. 2008, SPIE, 7012, 701227-1-701226-12

Lawrence, J. S. et al. 2009, Rev. Sci. Inst., 80, 064501-1-064501-10

Luong-Van, D. M. et al. 2008, SPIE, 7019, 70192U-1-70192U-10

Moore, A. et al. 2008, SPIE, 7012, 701226-1-701226-10

Yang, H. et al. 2009, PASP, 121, 174-184

Yuan, X. et al. 2008, SPIE, 7012, 70124G-1-70124G-8 